\title{
Analytic Modeling of Trench Waveguides for Channel Plasmon-Polariton Using Full-Hybrid Trial-Field Functions
}

\author{
Sujit Chattopadhyay ${ }^{1}$ and Pradip Kumar Saha ${ }^{2}$ \\ ${ }^{1}$ Indian Telecommunication Service, Kolkata 700001, India \\ ${ }^{2}$ Institute of Radio Physics and Electronics, University of Calcutta, 92, Acharya Prafulla Chandra Road, Kolkata 700009, India
}

Correspondence should be addressed to Pradip Kumar Saha, saha.pradipk@gmail.com

Received 17 November 2011; Accepted 18 December 2011

Academic Editor: Y. S. Kivshar

Copyright (C) 2012 S. Chattopadhyay and P. K. Saha. This is an open access article distributed under the Creative Commons Attribution License, which permits unrestricted use, distribution, and reproduction in any medium, provided the original work is properly cited.

\begin{abstract}
A full-hybrid trial-field modeling, which was proposed earlier by the authors for plasmonic structures of rectangular cross-section, has been applied to trench waveguides for channel plasmon polariton. Most of the computed results of complex propagation constant agree well with those obtained by method of lines and effective index method, though the latter two results themselves show some discrepancy. The particular aspect in which there is varying degree of disagreement among the results obtained in the above three methods is the imaginary part of the effective index seen by the plasmon-polariton.
\end{abstract}

\section{Introduction}

In the field of nanophotonics, the ability of surface plasmon polariton (SPP) to beat the diffraction limit is a tremendous boon to technology and its usefulness towards achieving higher integration density in photonic integrated circuits is now well established. As such, well-confined wave guidance and improved propagation length are the key aspects being addressed by the researchers. These goals in turn have led to a number of innovations in the SPP guiding structures. Reviews of SPP guides can be found, for example, in [1-3]. Apart from the basic SPP guiding structure of metal strip embedded in homogeneous or inhomogeneous dielectrics and its variants, gap-SPP and channel-SPP have been shown to have several desirable features [4-7]. Channel SPPs, commonly referred to as channel plasmon polariton or CPP, are guided by two basic structures. (a) The channel is a V-shaped groove in a metal. The supported CPP is generally referred to as V-groove channel plasmon polariton or V-groove CPP. (b) The channel is a narrow rectangular groove in a metal. The supported CPP is referred to as trench channel plasmon polariton or trench-CPP. Simultaneous availability of subwavelength lateral confinement and relatively long propagation length is the most desirable feature of CPP.
Photonic components based on both types of CPPs have been demonstrated in practice [8-10]. Together with different simulation techniques, the effective index method (EIM), traditionally employed in dielectric waveguide problems, has been used for determining the propagation characteristics of channel-SPP guides and in modeling SP waveguide components $[1,11,12]$. The main attractive feature of EIM is that the characteristics of $2 \mathrm{D}$ guides can be predicted by combining results obtained from analysis of $1 \mathrm{D}$ guides. Another method, the semianalytic method of lines, has proved to be a very useful tool for numerical modeling of plasmonic waveguides [13-16]. Minh and others [17] recently have applied MoL to calculate the propagation characteristics of trench-CPP waveguides and have compared their results with those obtained by EIM [12] and FDTD [18]. The present authors have carried out a simple analytic modeling of trench-CPP guides using full-hybrid trial-field functions. This approximate yet quite accurate technique was earlier successfully applied to metallic SPP guides of rectangular cross-section and to rectangular nanoholes in metals [19]. The computed values of complex effective index and propagation length for various trench dimensions and dielectric filling are compared with those in $[12,17]$. 


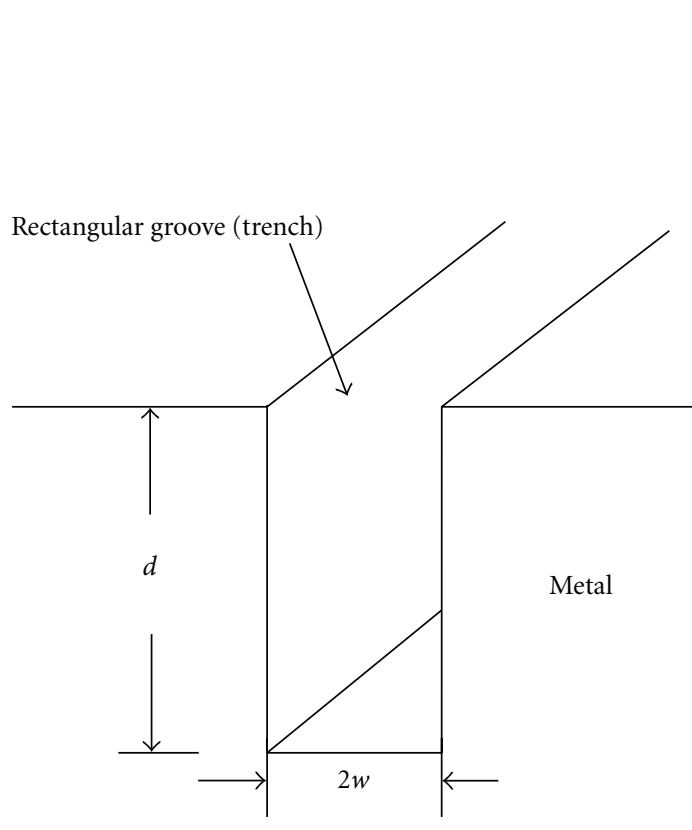

(a)

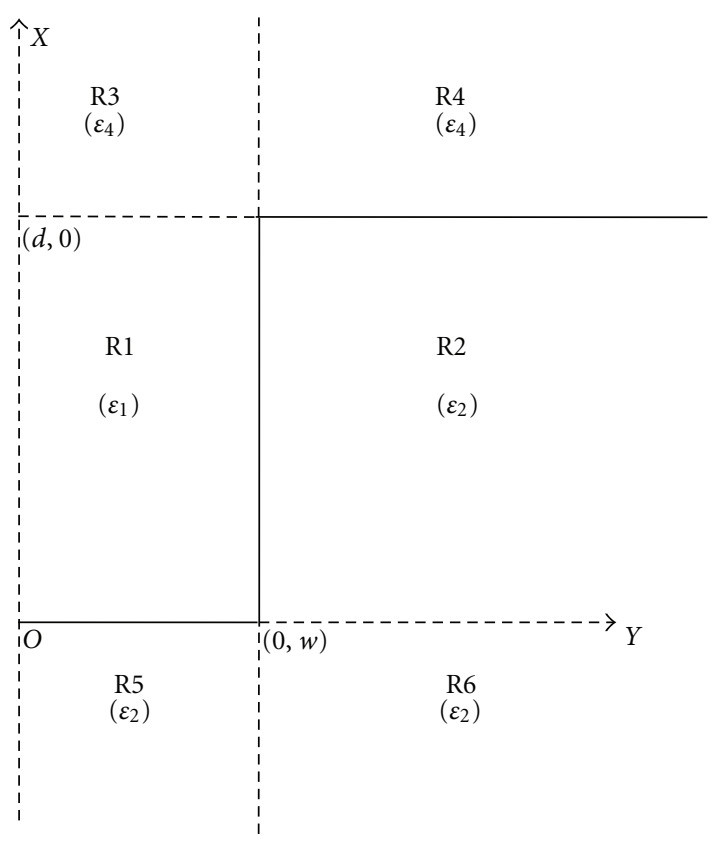

(b)

FIGURE 1: (a) Geometry of trench-CPP waveguide. (b) One half of trench-CPP guide segmented into six regions.

\section{Formulation of the Problem}

The geometry of the trench SPP guide is shown in Figure 1 (a). It is a rectangular groove of depth $d$ and width $2 w$ in a semi-infinite metal block. It can be bisected longitudinally by a symmetry plane at $y=0$. The groove is usually air-filled but can be filled with other dielectrics. All modal fields are assumed to have $\exp (-j \beta z)$ dependence in the direction of propagation.

The parameters are chosen as in $[12,17]$ : the wavelengths of operation $\lambda=1550 \mathrm{~nm}, 1033 \mathrm{~nm}, 775 \mathrm{~nm}$; the metal is gold with dielectric constant of $-131.95-\mathrm{j} 12.65$ at $1550 \mathrm{~nm}$; trench width $2 w=300 \mathrm{~nm}, 500 \mathrm{~nm}$; depth $d$ is variable.

2.1. Trial Field Modeling. In this approach, we would construct the trial transverse spatial functions for $\mathbf{E}_{\mathrm{y}}^{\mathrm{TE}}$ and $\mathbf{H}_{\mathrm{y}}^{\mathrm{TM}}$ fields corresponding to $\mathrm{TE}^{x}\left(E_{x}=0\right)$ and $\mathrm{TM}^{x}\left(H_{x}=0\right)$ modes, respectively, and derive all six field components of the hybrid plasmon modes of the structure from superposition of the $\mathrm{TE}^{x}$ and $\mathrm{TM}^{x}$ mode families [13]. While writing the trial field functions, first we consider the following points: (a) only one-half of the structure, shown in Figure 1(b), that takes into account the symmetry plane at $y=0$ is used for formulation, (b) the reduced structure is segmented into six regions, R1 to R6, as shown in Figure 1(b), (c) trial fields for each region $i$ ( $i=1$ to 6 ) are constructed separately such that each trial field $\Psi_{i}$ satisfies the Helmholtz equation in respective region $i$, and (d) continuity of the transverse wavevector at any interface between two media is also assumed. Secondly, since the success of trial-field modeling depends on how well the trial functions approximate the true fields, in writing the trial field functions inside the trench (region
R1), the nature of field variation, particularly with depth, as reported in $[12,17]$ was taken into consideration. Clearly, the symmetry plane at $y=0$ is electric for the dominant field configuration. Provision for magnetic symmetry, however, is retained in the formulation. For $x$-dependence of the field inside the trench, with respect to the chosen coordinates, one has the option of writing the functional form as $\sinh k_{x} x$ or $\cosh k_{x} x$, both growing toward the top of the trench and being almost identical beyond the argument value of about two. Choice of sinh function forces the main electric field $E_{y}$ to be zero at the trench bottom irrespective of the trench depth. The reported variation $[12,17]$, however, shows that the field is always very small (but not zero) at the bottom, and this value depends on the depth $d$. For short depths, the field at the bottom is not negligibly small and penetration into the metal is also significant. For deep trenches, both are very small. In fact, our computation with sinh function in the trial field for different trench widths always yielded the propagation constant of the gap-SPP mode [12]. Thus cosh dependence on $x$ inside the trench appears to be the logical choice for the trial field function. On inspection and with the above considerations in mind, the trial-field functions for different regions are written as follows. Although regions R5 and R6 are shown in the segmented structure, the trial fields in these two regions are not written. Obviously, field matching at the R2-R6 and R5-R6 interfaces are not meaningful. Similar observations can also be made about field matching at R1-R3 and R3R4 interfaces. Furthermore, field matching at the trench bottom (R1-R5 interface) is not necessary because it does not yield any useful information. This is to be expected from the reported variation of field with depth [12], which 
shows that this interface is not plasmonic; that is, it does not support a surface electromagnetic wave. So finally we write the trial field functions only for three regions R1, R2, and R4:

$$
\begin{aligned}
E_{1 y}^{\mathrm{TE}} & =\sum_{n=1}^{\infty} A_{1 n} \cosh \left(k_{n 1 x} x\right){ }_{\cosh }^{\sinh }\left(k_{n 1 y} y\right), \\
H_{1 y}^{\mathrm{TM}} & =\sum_{n=1}^{\infty} B_{1 n} \cosh \left(k_{n 1 x} x\right)_{\sinh }^{\cosh }\left(k_{n 1 y} y\right), \\
E_{2 y}^{\mathrm{TE}} & =\sum_{n=1}^{\infty} A_{2 n} \cosh \left(k_{n 1 x} x\right) e^{-k_{n 2 y}(y-w),} \\
H_{2 y}^{\mathrm{TM}} & =\sum_{n=1}^{\infty} B_{2 n} \cosh \left(k_{n 1 x} x\right) e^{-k_{n 2 y}(y-w)}, \\
E_{4 y}^{\mathrm{TE}} & =\sum_{n=1}^{\infty} A_{4 n} e^{-k_{n 4 x}(x-d)} e^{-k_{n 2 y}(y-w)}, \\
H_{4 y}^{\mathrm{TM}} & =\sum_{n=1}^{\infty} B_{4 n} e^{-k_{n 4 x}(x-d)} e^{-k_{n 2 y}(y-w)} .
\end{aligned}
$$

The transverse wave numbers are related to $\beta$ by the following equation:

$$
k_{n i x}^{2}+k_{n i y}^{2}=\beta^{2}-k_{0}^{2} \varepsilon_{i} .
$$

The upper and lower functions correspond, respectively, to magnetic and electric walls at the symmetry plane $y=0$. The expressions (1) for the field components need some clarifications. The summation on $n$ in expressions (1) is for the purpose of writing the trial functions in generalized form and do not represent expansion in terms of orthogonal basis functions. Only the nature of a physical solution for a prospective plasmon mode is envisaged while constructing the trial functions. However, the eigenvalue equations are transcendental in nature and can be solved for a series of complex roots $\left(k_{\text {nix }}, k_{\text {niy }}\right)$ which would give the same value of $\beta$ for a particular mode and different $\beta$ for different modes. Therefore, it is sufficient to compute, say, $k_{11 x}$ and $k_{11 y}$, for computation of $\beta$ of the lowest mode. However, the root set $\left(k_{\text {nix }}, k_{\text {niy }}\right)$ can be computed to obtain the field profiles in different regions.

2.2. Eigenvalue Equations. Once all six field components in each region (R1, R2, R4) are derived from the trial fields, the next task would be to apply the continuity conditions. It is not necessary to impose all the boundary conditions at all the interfaces which only overdefines the problem. We found that application of continuity of tangential field components at only the R1-R2 and R2-R4 interfaces (between two different media) yields the desired eigenvalue equations, given by (3), for all values of $n$ :

$$
\operatorname{Det}\left[\begin{array}{cccc}
\cosh \left(k_{n 1 x} d\right) & -\frac{k_{n 1 x} k_{n 2 y}}{\omega \beta \varepsilon_{0} \varepsilon_{2}} \sinh \left(k_{n 1 x} d\right) & -1 & -\frac{k_{n 4 x} k_{n 2 y}}{\omega \beta \varepsilon_{0} \varepsilon_{4}} \\
\frac{k_{n 1 x} k_{n 2 y}}{\omega \beta \mu_{0}} \sinh \left(k_{n 1 x} d\right) & \cosh \left(k_{n 1 x} d\right) & \frac{k_{n 4 x} k_{n 2 y}}{\omega \beta \mu_{0}} & -1 \\
\frac{k_{n 2 y}}{\beta} \cosh \left(k_{n 1 x} d\right) & -\frac{k_{n 1 x}}{\omega \varepsilon_{0} \varepsilon_{2}} \sinh \left(k_{n 1 x} d\right) & -\frac{k_{n 2 y}}{\beta} & -\frac{k_{n 4 x}}{\omega \varepsilon_{0} \varepsilon_{4}} \\
\frac{k_{n 1 x}}{\omega \mu_{0}} \sinh \left(k_{n 1 x} d\right) & \frac{k_{n 2 y}}{\beta} \cosh \left(k_{n 1 x} d\right) & \frac{k_{n 4 x}}{\omega \mu_{0}} & -\frac{k_{n 2 y}}{\beta}
\end{array}\right]=0,
$$

where $X_{12}=\left(k_{n 1 x}^{2}+k_{0}^{2} \varepsilon_{1}\right) /\left(k_{n 1 x}^{2}+k_{0}^{2} \varepsilon_{2}\right)$.

\section{Results of Computation}

With the chosen parameters as mentioned in Section 2, complex effective index and propagation length from its imaginary part were computed and are presented in Figures 2(a) to $4(\mathrm{~b})$.

3.1. Effect of Trench Depth. The variation of $\operatorname{Re}\left(n_{\mathrm{eff}}\right)$ with trench depth $d$, as shown in Figure 2(a), is very much similar to the EIM results [12], though our computed values are always slightly higher. The computed propagation length $L$ (Figure 2(b)) for $2 w=300 \mathrm{~nm}$ is almost same as that in [12]. For $2 w=500 \mathrm{~nm}$ our values, however, are slightly lower, particularly at smaller depths. Though we have not shown comparison with MoL data in Figures 2(a) and 2(b); an examination of Figures 3(a) and 3(b) of [17] indicate that our data of $\operatorname{Re}\left(n_{\text {eff }}\right)$ agree better with the MoL data than with the EIM data. However, our computed values of propagation length agree much better with EIM data. The MoL value of propagation length hardly changes with trench depth and shows a small increase at small trench depth of about $1 \mu \mathrm{m}$. The discrepancy in the nature of variation of the MoL values of $L$ with respect to EIM data for small trench depths has been noted in [17]. All the previously reported data and our results confirm that subwavelength lateral confinement as well as relatively long propagation length can be achieved with trench-CPP waveguides with deep grooves. Specifically, the fundamental trench-CPP mode 


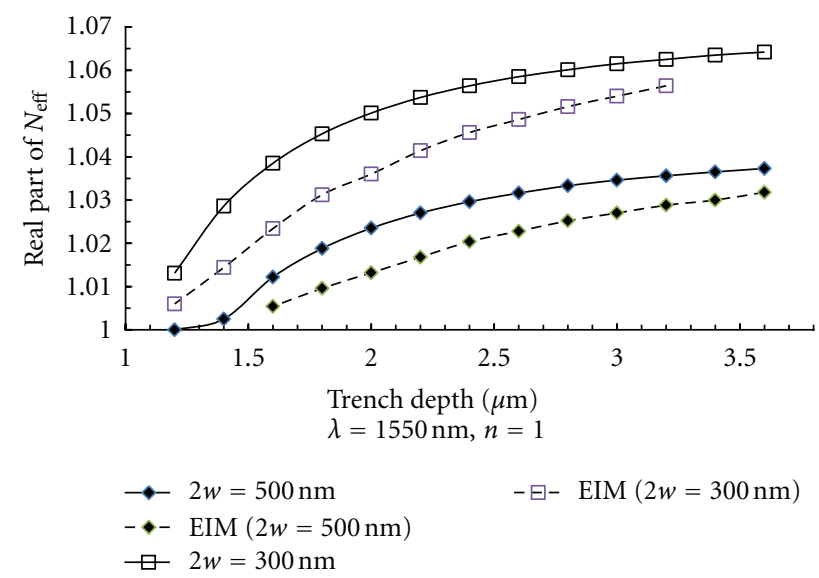

(a)

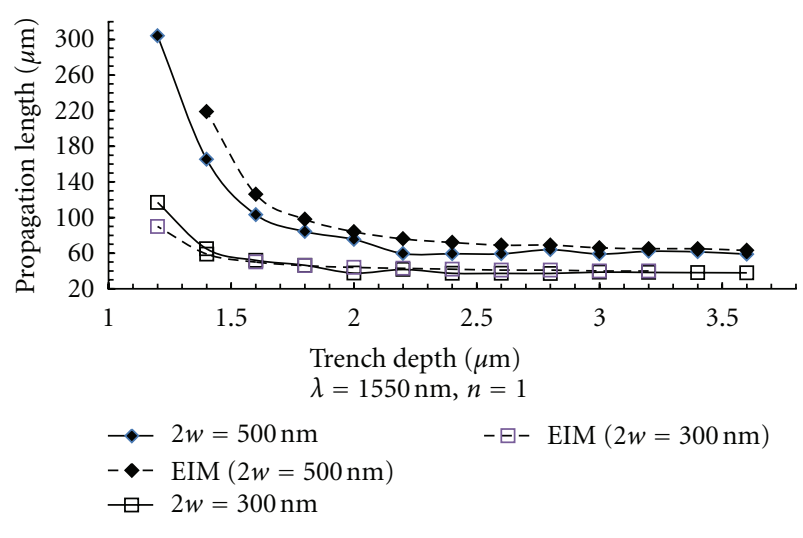

(b)

Figure 2: (a) Dispersion of the real part of the fundamental trench-CPP mode effective index with depth of the air-filled trench. (b) Dispersion of the propagation length of the fundamental trench-CPP mode with depth of the air-filled trench.

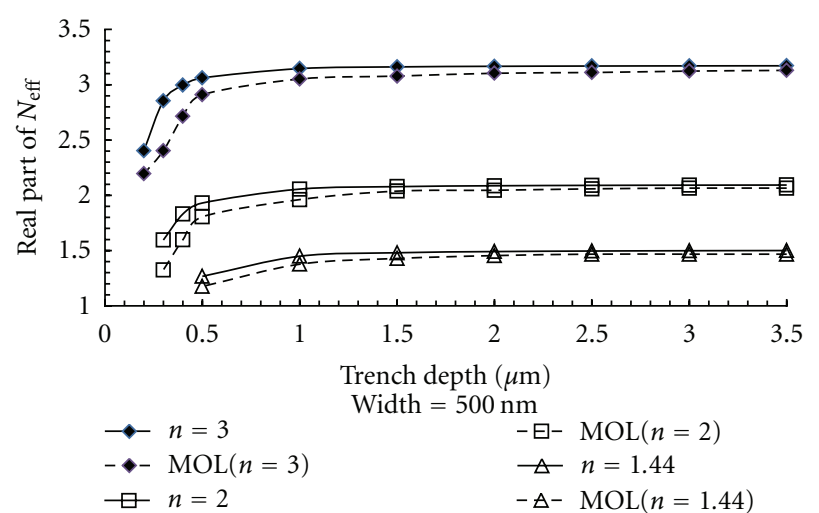

(a)

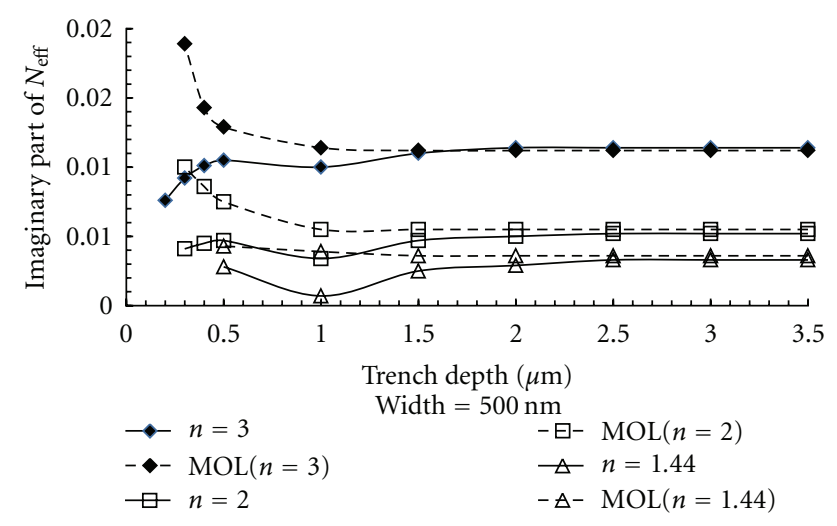

(b)

FIGURE 3: (a) Dispersion of the real part of the fundamental trench-CPP mode effective index with trench depth for different dielectric inserts inside the trench $(n=3,2,1.44)$. (b) Dispersion of the imaginary part of the fundamental trench-CPP mode effective index with trench depth for different dielectric inserts inside the trench $(n=3,2,1.44)$.
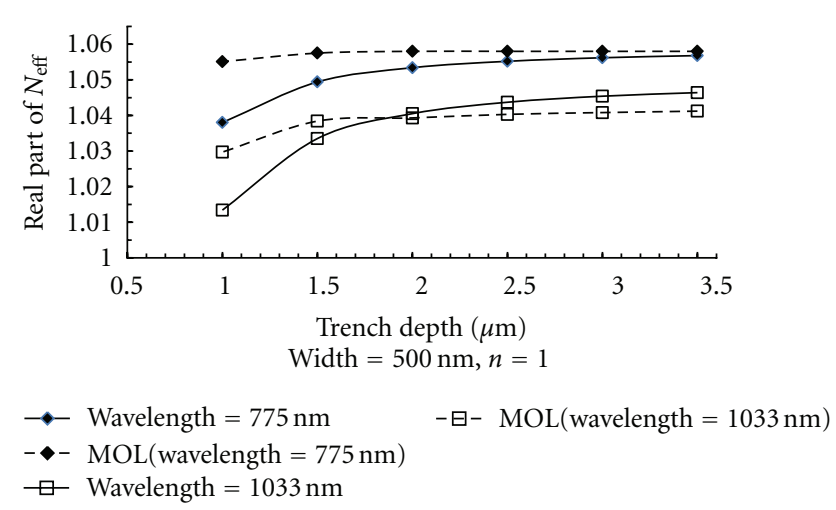

(a)

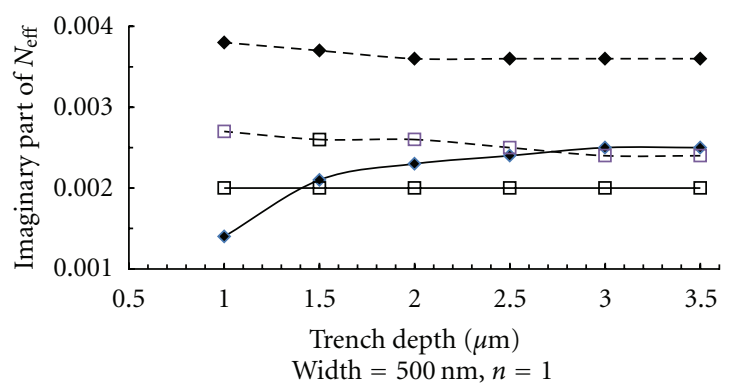

$\square-$ Wavelength $=775 \mathrm{~nm} \quad-\bullet-\operatorname{MOL}($ wavelength $=1033 \mathrm{~nm})$
$-\bullet-$ MOL $($ wavelength $=775 \mathrm{~nm})$
$\square$ Wavelength $=1033 \mathrm{~nm}$

(b)

FIGURE 4: (a) Dispersion of the real part of the fundamental trench-CPP mode effective index for different wavelengths of operation with depth of the air-filled trench. (b) Dispersion of the imaginary part of the fundamental trench-CPP mode effective index for different wavelengths of operation with depth of the air-filled trench. 
which shows these features require large depth-to-width ratio.

3.2. Effect of Dielectric Filling of Trench. It has been shown $[12,17]$ that, in a trench waveguide with air-filled groove (which can be looked upon as two right-angled wedges hybridized by a finite-depth rectangular groove), the field is mainly confined at the two corners at the top of the trench. In [17] it was proposed that dielectric filling $\left(\varepsilon_{1}>1\right)$ of the groove can be utilized for stronger confinement of field inside the trench. Results of MoL computation have established the projected improvement in normalized phase constant. Unfortunately, this is accompanied by increase in normalized attenuation constant. However, there is still provision for tradeoff between the two characteristics obtained with dielectric filled trench.

The trial-field modeling with segmented problem area is well suited for computing the complex effective index of dielectric-filled trench configuration. The results of our computation are presented in Figures 3(a) and 3(b), where comparison with MoL data also is shown. The agreement of $\operatorname{Re}\left(n_{\text {eff }}\right)$ is excellent. However, there is discrepancy in the imaginary part particularly for the lower trench depths $(<1.5 \mu \mathrm{m})$, which is just the opposite of the variation obtained from MoL for air-filled trench. In [17], $\operatorname{Im}\left(n_{\text {eff }}\right)$ increases with decreasing trench depth which is just the opposite of the variation obtained by MoL for air-filled trench. It is difficult to understand why dielectric filling should cause this reversal in the nature of variation. The authors in [17] also have not offered any plausible explanation. The results obtained from trial-field modeling indicate the $\operatorname{Im}\left(n_{\text {eff }}\right)$ decreases with decreasing trench depth for both air- and dielectric-filled grooves. The decrease, however, is not exactly monotonic when $\varepsilon_{1}>1$. For small depths of both air- and dielectric-filled grooves, the confinement is poor, the loss is lower, and hence the propagation length would be longer.

3.3. Effect of Wavelength. Figures 4(a) and 4(b) show the computed effective index for air-filled trench as function of depth at two other (smaller) wavelengths $775 \mathrm{~nm}$ and $1033 \mathrm{~nm}$. These are compared with the MoL data [17]. The agreement of $\operatorname{Re}\left(n_{\text {eff }}\right)$ data is reasonably good except at small trench depths where our results decrease faster. Clearly, the wave confinement improves at lower wavelengths. Our computed values of $\operatorname{Im}\left(n_{\text {eff }}\right)$ at these wavelengths predict longer propagation lengths than the MoL results suggest. In fact our computed values of $L$ at $1550 \mathrm{~nm}$, which we have already mentioned to be in better agreement with the EIM data, also indicated higher propagation length than the MoL prediction.

\section{Conclusions}

A simple and approximate yet reasonably accurate method of modeling based on full-hybrid trial field functions has been applied to trench-CPP waveguide. The technique was earlier validated by successfully applying it to metal-strip SPP guide and nanoholes of rectangular cross-section in metals [19]. The literature on trench-CPP is very much limited at this time. The reported comparison of EIM and MoL results showed only partial agreement $[12,17]$. And now, our results, obtained with trial-field modeling, agree well in parts with EIM and partly with MoL data. The problem of discrepancy appears to be mainly in the imaginary part of the effective index or the propagation length, particularly for small values of trench depth. As such any claim about one particular method being more accurate than the others may not be justified at this point of time. Perhaps more detailed study with more rigorous formulation will resolve this issue. Meanwhile we put forward our approach as a very much useful method.

\section{References}

[1] P. Berini, "Long-range surface plasmon polaritons," Advances in Optics and Photonics, vol. 1, no. 3, pp. 484-588, 2009.

[2] S. I. Bozhevolnyi, Ed., Plasmonic Nanoguides and Circuits, Pan Standford, Singapore, 2009.

[3] V. M. Shalaev and S. Kawata, Eds., Nanophotonics with Surface Plasmons, Elsevier, Amsterdam, The Netherlands, 2007.

[4] J. Q. Lu and A. A. Maradudin, "Channel plasmons," Physical Review B, vol. 42, no. 17, pp. 11159-11165, 1990.

[5] I. V. Novikov and A. A. Maradudin, "Channel polaritons," Physical Review B, vol. 66, no. 3, Article ID 035403, 13 pages, 2002.

[6] S. I. Bozhevolnyi, V. S. Volkov, E. Devaux, and T. W. Ebbesen, "Channel plasmon-polariton guiding by subwavelength metal grooves," Physical Review Letters, vol. 95, no. 4, Article ID 046802, 4 pages, 2005.

[7] S. I. Bozhevolnyi, V. S. Volkov, E. Devaux, J. Y. Laluet, and T. W. Ebbesen, "Channelling surface plasmons," Applied Physics A, vol. 89, no. 2, pp. 225-231, 2007.

[8] D. K. Gramotnev and D. F. P. Pile, "Single-mode subwavelength waveguide with channel plasmon-polaritons in triangular grooves on a metal surface," Applied Physics Letters, vol. 85, no. 26, pp. 6323-6325, 2004.

[9] S. I. Bozhevolnyi, V. S. Volkov, E. Devaux, J. Y. Laluet, and T. W. Ebbesen, "Channel plasmon subwavelength waveguide components including interferometers and ring resonators," Nature, vol. 440, no. 7083, pp. 508-511, 2006.

[10] V. S. Volkov, S. I. Bozhevolnyi, E. Devaux, and T. W. Ebbesen, "Compact gradual bends for channel plasmon polaritons," Optics Express, vol. 14, no. 10, pp. 4494-4503, 2006.

[11] A. Boltasseva, T. Nikolajsen, K. Leosson, K. Kjaer, M. S. Larsen, and S. I. Bozhevolnyi, "Integrated optical components utilizing long-range surface plasmon polaritons," IEEE Journal of Lightwave Technology, vol. 23, no. 1, pp. 413-422, 2005.

[12] S. I. Bozhevolnyi, "Effective-index modeling of channel plasmon polaritons," Optics Express, vol. 14, no. 20, pp. 94679476, 2006.

[13] P. Berini, "Plasmon-polariton waves guided by thin lossy metal films of finite width: bound modes of symmetric structures," Physical Review B, vol. 61, no. 15, pp. 10484-10503, 2000.

[14] P. Berini, "Plasmon-polariton waves guided by thin lossy metal films of finite width: bound modes of asymmetric structures," Physical Review B, vol. 63, no. 12, Article ID 125417, 15 pages, 2001.

[15] H. A. Jamid and M. N. Akram, "A new higher order finitedifference approximation scheme for the method of lines," 
IEEE Journal of Lightwave Technology, vol. 19, no. 3, pp. 398404, 2001.

[16] S. Chattopadhyay and P. K. Saha, "Computation of surface plasmon modes in prism-buffer layer-metal configuration using a method of lines modeling scheme," in Proceedings of the IEEE Applied Electromagnetics Conference, pp. 1-4, Kolkata, India, December 2009.

[17] T. T. Minh, K. Tanaka, and M. Tanaka, "Analysis propagation characteristics of the surface plasmon polariton trench waveguides by method of lines," in Proceedings of the Progress in Electromagnetics Research Symposium, pp. 555-559, Cambridge, Mass, USA, July 2010.

[18] S. H. Chang, T. C. Chiu, and C. Y. Tai, "Propagation characteristics of the supermode based on two coupled semi-infinite rib plasmonic waveguides," Optics Express, vol. 15, no. 4, pp. 1755-1761, 2007.

[19] S. Chattopadhyay and P. K. Saha, "Study of bound surface plasmon modes on subwavelength structures of rectangular cross section using a simple analytical technique," Journal of Optics A, vol. 10, no. 9, Article ID 095201, 2008. 

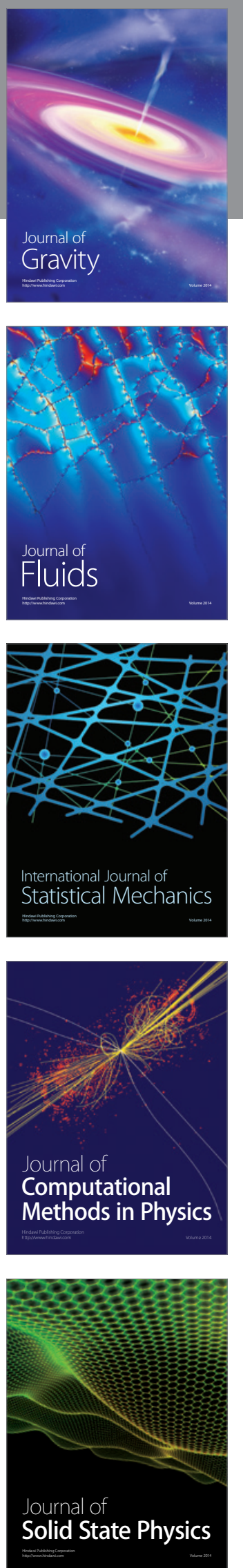
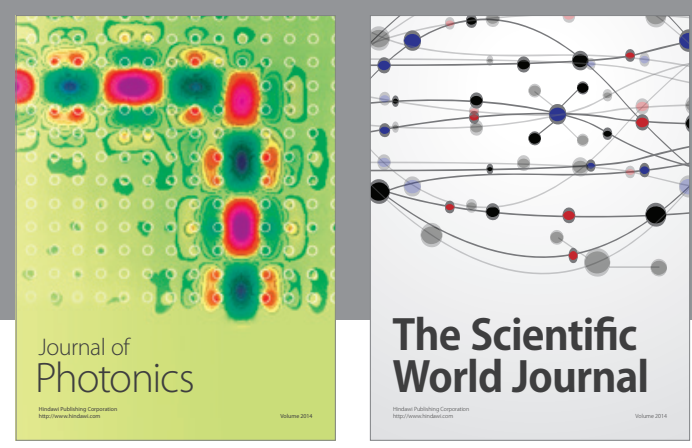

The Scientific World Journal

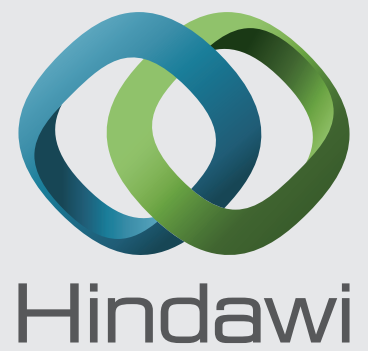

Submit your manuscripts at http://www.hindawi.com
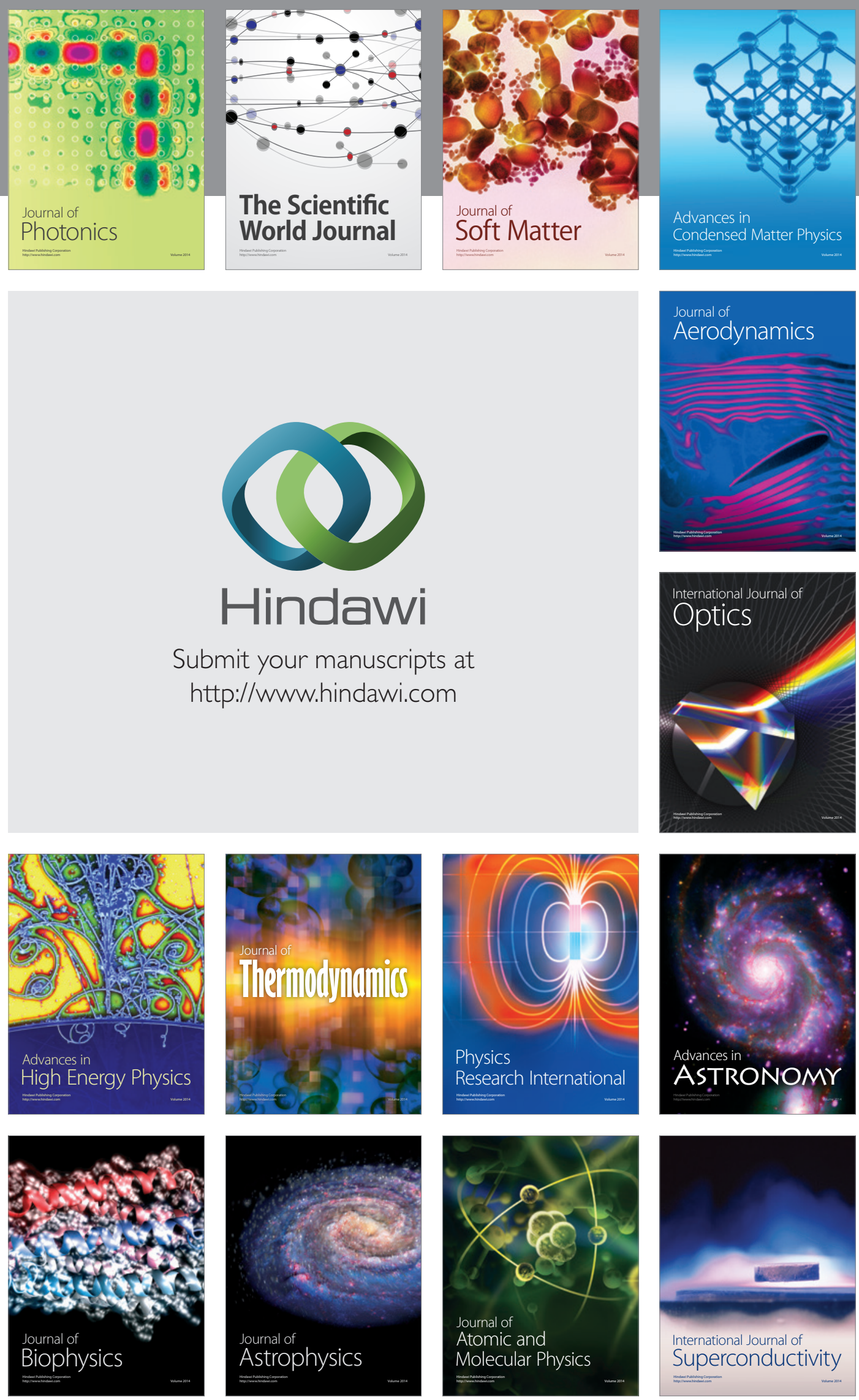
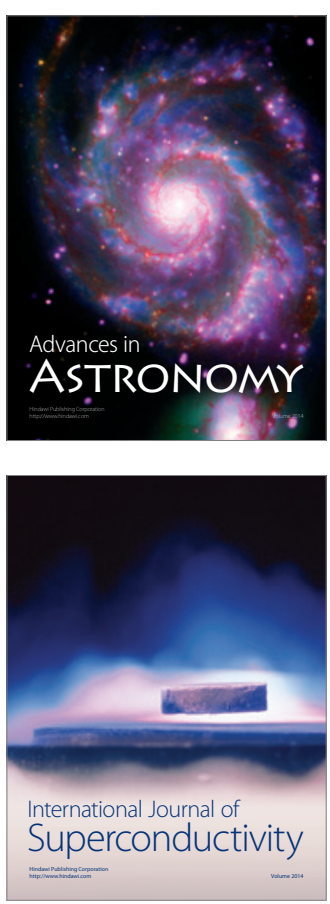